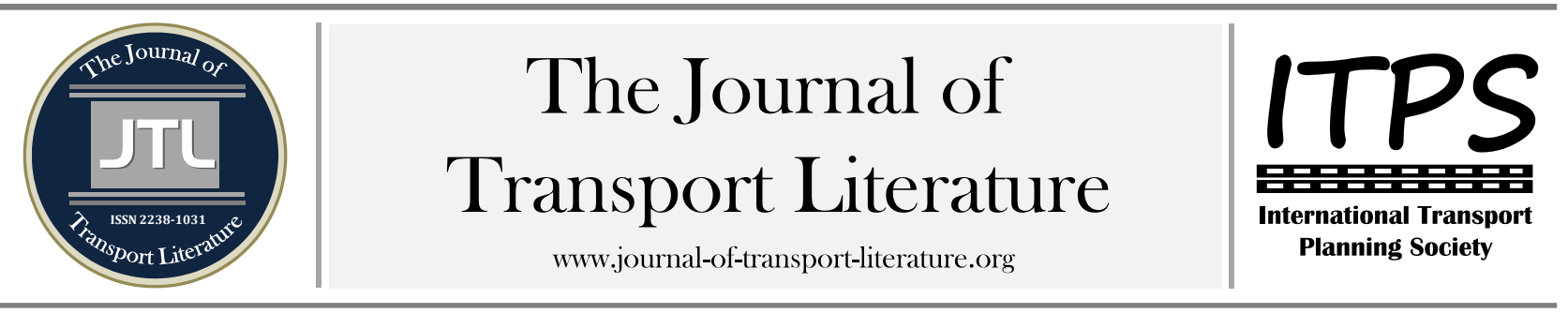

\title{
Detecção de padrões de mobilidade urbana em dia atípico a partir de sistema de bilhetagem eletrônica: análise das viagens na RMRJ durante o carnaval
}

\author{
Gabriel T. de Oliveira1; Alan Jeferson de Oliveira da Silva ${ }^{2,+}$; Carlos David Nassi ${ }^{3}$ \\ 1 Universidade Federal do Rio de Janeiro, Rio de Janeiro, Brasil \\ 2 Centro Universitário UNIABEU, Belford Roxo, Brasil
}

3 Universidade Federal do Rio de Janeiro, Rio de Janeiro, Brasil

\section{Article Info}

Palavras-chave:

SBE

Demanda

Mobilidade Urbana

BUE

Transporte Coletivo

Submitted 3 Jul 2015;

received in revised form $5 \mathrm{Jul} 2015$;

accepted 12 Jul 2015

\section{Licensed under}

Creative Commons

CC-BY 3.0 BR.

\section{Resumo}

Este artigo foca na análise de padrões de mobilidade urbana durante os dias de carnaval da cidade do Rio de Janeiro a partir de dados do Sistema de Bilhetagem Eletrônica do programa de subsídio ao transporte de passageiros do Governo do Estado do Rio de Janeiro, o chamado Bilhete Único Estadual. Os padrões em termos de quantidade de transações, de distribuição modal e horária e de distribuição espacial da origem são analisados de forma comparativa àqueles encontrados em dias típicos de semana e de fim de semana. 0 estudo conclui que a utilização de dados do Sistema de Bilhetagem Eletrônica permite aos agentes públicos responsáveis e às operadoras a melhor elaborar esquemas operacionais para grandes eventos como o carnaval. Recomenda-se para tanto, que o SBE seja de fato único entre todos os modos e categorias tarifárias.

+ Corresponding author. Universidade Federal do Rio de Janeiro, Programa de Engenharia de Transportes.PET - COPPE / UFRJ. Ilha do Fundão. 21945970 - Rio de Janeiro, RJ - Brasil.

E-mail address: alan@pet.coppe.ufrj.br.

\section{Introdução}

A Região Metropolitana do Rio de Janeiro - RMRJ com 12,0 milhões de habitantes (IBGE, 2015) é a segunda maior do Brasil em termos de população, mas a primeira em utilização do transporte público. De acordo com o Plano Diretor de Transportes Urbanos, das viagens motorizadas, $71,5 \%$ - ou cerca de 11,0 milhões de viagens diariamente - são realizadas no sistema de transporte público existente (SETRANS-RJ, 2014). Diversos estudos (Zoghi, Siamardi, \& Tolouei, 2009; Giuliano, 1985) já evidenciaram que os movimentos diários da população são afetados por eventos sazonais e pontuais. Normalmente, ocorrem mudanças significativas nas atividades e motivações da população e consequentemente no padrão de viagens. Este é o caso em meses de férias escolares, feriados e grandes eventos. 0 carnaval é um exemplo de grande evento que suscita curiosidade de estudo. Na cidade do Rio de Janeiro segundo a Riotur, aproximadamente 1 milhão de pessoas viram os desfiles no sambódromo e 5 milhões pularam o carnaval nos blocos de rua da cidade (RIOTUR-RJ, 2015). Por se repetir anualmente, diversas já são os esquemas específicos promovidos para adequar o sistema de transporte de passageiros às mudanças de demanda. De fato, frente às limitações da cidade em relação à infraestrutura de mobilidade urbana e a sua operação, os grandes eventos exigem dos órgãos competentes medidas para mitigar os impactos gerados no sistema viário e na mobilidade dos usuários nas estreitas horas de pico. Mas, métodos relativamente envolvendo sistemas automáticos de coleta de dados existem para dar poder aos agentes públicos no planejamento de esquemas operacionais específicos (DUNN, 2009).

O Governo do Estado do Rio de Janeiro implantou, desde 2010, um programa de subsídio ao transporte de passageiros na Região Metropolitana do Rio de Janeiro. 0 chamado Bilhete Único Estadual - BUE permite ao usuário, através de um cartão, usufruir de um benefício tarifário em diversas linhas intermunicipais e na integração entre dois veículos numa mesma viagem. No ano de 2014 foram realizadas 401 milhões de transações neste sistema. Cada transação, por estar vinculada a um cartão, compõe um quebra-cabeça que permite estimar os deslocamentos de seus usuários no sistema de transporte público. Estes dados são assim uma fonte de informação importante para os agentes do poder público que são capazes de entender, em tempo real, como se deslocam as pessoas e assim poder planejar esquemas operacionais que melhor se adequem aos usuários (MUNIZAGA, PALMA e FISCHER, 2011.).

Este trabalho foi desenvolvido com o objetivo geral de analisar o padrão de viagem do usuário do programa de Bilhete Único Estadual durante os dias de carnaval. Para tanto são analisadas a quantidade, a distribuição modal, a distribuição horária e a distribuição de origem das transações realizadas neste Sistema de Bilhetagem Eletrônica. As análises são traçadas de modo comparativo com os padrões observados em dias típicos de semana e de fim de semana. 0 trabalho é dividido da seguinte forma: na seção 1 é realizada uma revisão bibliográfica sobre trabalhos correlatos e é apresentada a Metodologia empregada para obtenção de dados e para análise; na seção 2 os Resultados obtidos em relação ao padrão de viagem nos dias de carnaval são apresentados e discutidos; e na seção 3 é traçada uma conclusão sobre o trabalho e suas limitações e possibilidades de desdobramentos em pesquisas futuras.

\section{Revisão Bibliográfica}

À gama de tecnologias empregadas para processar os dados de viagens (transações) para fins de arrecadação tarifária se dá o nome de Sistema de Bilhetagem Eletrônica - SBE (ZHAO, 2007). Do ponto de vista do usuário, o cartão eletrônico é o meio mais adotado para realização das transações neste tipo de sistema, se comparado a outros como o cartão magnético (GUERRA, BARBOSA e DE OLIVEIRA, 
2014; PELLETIER, TRÉPANIER e MORENCY, 2010). O programa de benefício tarifário intitulado de Bilhete Único Estadual - BUE foi instituído em 2010 na Região Metropolitana do Rio de Janeiro pelo Governo do Estado do Rio de Janeiro. Esse programa tem enorme relevância socioeconômica para RMRJ (SILVA, NASSI E MURTA, 2012). Para viabilização da política tarifária, foi adotado o Sistema de Bilhetagem Eletrônica. 0 volume de dados gerados por este sistema possibilita variados estudos e análises sobre a sua dinâmica de utilização e sobre as interações espaciais na região (SILVA, 2013). Diversos autores já estudaram as vantagens e desvantagens da utilização do SBE, tanto para fins de arrecadação tarifária (PELLETIER, TRÉPANIER e MORENCY, 2010), quanto para aplicação em planejamento de transportes (MUNIZAGA, PALMA e FISCHER, 2011; ZHAO, 2007; GUPTA, 2006). Em relação à detecção de padrões, foco deste artigo, alguns autores já empreenderam estudos sobre a regularidade e variabilidade temporal e locacional para diferentes categorias de usuários na escala semanal e sazonal (CHU, SPURR e CHAPLEAU, 2013; LIU, HOU, et al., 2009; MORENCY, TRÉPANIER e AGARD, 2007). Este artigo inova ao tentar detectar diferenças de padrão entre dias festivos (de Carnaval), dias típicos e de fim de semana por meio da utilização de dados de SBE.

\section{Metodologia}

Para análise dos padrões de viagens da Região Metropolitana do Rio de Janeiro a partir dos dados do Bilhete Único Estadual, foram adotadas as seguintes etapas: I. Prospecção de Dados do SBE; II. Identificação par O-D das linhas que compõem o SBE; III. Alocação das viagens por sentido a partir de dados do PDTU; IV. Separação e representação das origens de viagens por município da RMRJ de origens de viagens por município da RMRJ. A seguir as etapas são descritas:

\section{I - Prospecção de Dados do SBE.}

O Sistema de Bilhetagem Eletrônica - SBE do BUE conta com diversos dados sobre as transações realizadas por cartões habilitados para o benefício do Bilhete Único. Para efeito deste trabalho, foram prospectados a quantidade de transações realizadas para cada linha do SBE e em cada data e hora dos dias analisados (ex: linha de código '07269', data '2015-02-15', '09' horas, '38' transações). Com esta etapa foi possível descrever a quantidade de transações acontecendo por dia em um dia típico, em um dia de fim de semana e em um dia de carnaval; a distribuição modal observada e a distribuição de transações por horário do dia. Para a análise da origem das transações para cada município da RMRJ, foram necessários ainda a realização das etapas subsequentes.

\section{II - Identificação par O-D das linhas que compõem o SBE.}

A linha conta, no SBE, com um código único de identificação, com sua vista e número cadastral (ex: código '07269', número '440B', vista 'Queimados x Central'). Para a identificação da origem e destino das linhas que compõem o SBE, foi realizada uma identificação do município de origem e destino das mesmas a partir do nome da vista. No exemplo acima, o município de origem foi identificado como 'Queimados' e o município de destino foi identificado como 'Rio de Janeiro'. Esta identificação foi realizada para 535 linhas de ônibus intermunicipais e 408 linhas de vans intermunicipais a partir de uma conferência das linhas georreferenciadas e quando indisponível a partir de uma consulta ao cadastro em sindicatos e em ferramentas de busca. Para 1001 linhas municipais, foram identificados apenas o município de localização das mesmas, denominado neste processo como o município de origem. A mesma lógica foi utilizada para as estações de trens, metrôs, barcas e BRTs, que se encontram cadastradas no SBE como linhas. A tabela 1 é um exemplo do resultado da identificação.

\begin{tabular}{rcrrr}
\multicolumn{5}{c}{ Tabela 1 - Identificação de par 0-D das linhas que compõem o SBE } \\
\hline Nome da Vista & Modo & Abran-gência & Município de Origem & Município de Destino \\
\hline Queimados x Central & Ônibus & I & Queimados & Rio \\
Acari x Fazenda Botafogo & Ônibus & $\mathrm{M}$ & Rio & - \\
Saracuruna & Trem & $\mathrm{M}$ & Duque de Caxias & \\
Cantagalo & Metrô & $\mathrm{M}$ & Rio \\
\hline
\end{tabular}

\section{III - Alocação das viagens por sentido a partir de dados do PDTU.}

Uma vez as linhas intermunicipais com par origem e destino alocados, foi necessário distribuir a totalidade de transações para cada um dos dois sentidos possíveis (município $1 \rightarrow$ município 2 e município $2 \rightarrow$ município 1 ). Para a maioria das linhas, há uma grande concentração do fluxo de passageiros em um sentido. Porém, alocação da totalidade de viagens em apenas um dos sentidos possíveis não corresponde à realidade. Para chegar à distribuição entre cada sentido possível de um par de município, primeiramente foram consultados os dados de Origem e Destino da revisão do Plano Diretor de Transportes Urbanos de 2011 (SETRANS, 2013). O PDTU descreve a distribuição de viagens entre cada sentido para o pico da manhã e para o pico da tarde. Para os outros horários do dia foi adotada a média aritmética das distribuições de pico. Seguindo o exemplo anterior, foi detectado que, no pico da manhã, o sentido 'Queimados' $\rightarrow$ 'Rio de Janeiro' corresponde a $80 \%$ das viagens e 'Rio de Janeiro' $\rightarrow$ 'Queimados' corresponde à $20 \%$. Para as transações nas linhas municipais, tanto a origem quanto o destino da totalidade de viagens foi considerado ocorrendo dentro do próprio município de abrangência da linha. Ao final desta etapa, chegou-se à quantidade de transações com origem em cada um dos municípios da RMRJ.

\section{IV - Separação e representação das origens de viagens por município da RMRJ.}

Com a alocação da origem de cada linha, dia e horário, foi realizado um somatório do total de transações para cada um dos municípios da RMRJ. Para análise das origens, foram separados apenas os municípios da RMRJ que não a capital. Esta medida teve como objetivo evitar possíveis distorções na análise visto que o Rio de Janeiro possui uma quantidade expressivamente maior de transações em comparação com os outros municípios da RMRJ. Para a produção do mapa das transações com BUE por origem de município nos dias de carnaval foi gerado um gráfico usando o método matemático de Quebra Natural no software ArcGIS $10.1 \mathrm{com}$ os dados de quantidade de origem de transações para cada um dos municípios da RMRJ excluindo o Rio de Janeiro. Esse método identifica pontos de quebra entre as classes por meio de algoritmo estatístico que se fundamenta na variabilidade dos dados que torna mínima a soma da variância dentro de cada uma das classes. As classes são assim divididas onde existe relativamente grandes saltos nos valores dos dados.

\section{Resultados e Discussão}

Nesta seção são apresentados os resultados da análise sobre os dados prospectados do SBE do BUE. Os resultados são discutidos na seguinte ordem: quantidade de transações, distribuição modal, distribuição horária e distribuição da origem.

\subsection{Quantidade de Transações}

Na RMRJ em um dia típico ocorrem em média quase 1,2 milhões de viagens no sistema BUE. No Carnaval de 2015, a média foi de 392 milhões de viagens por dia, valor correspondente a $33 \%$ do total de viagens em um dia típico e bem semelhante à quantidade de viagens em um dia de fim de semana (384 milhões de viagens). Considerando a possibilidade do usuário de realizar uma integração com o BUE, a estas viagens correspondem uma quantidade maior de transações. 
Tabela 2. Média de Viagens e Transações na RMRJ no Sistema de Bilhete Único Estadual

$\begin{array}{llll}\text { Dia } & \text { Média de Viagens por Dia } & \text { \% de um Dia Típico } & \text { Média de Transações por Dia } \\ \text { Carnaval } & 392.140,75 & 33 \% & 602.252,75 \\ \text { Fim de Semana } & 384.012,50 & 32 \% & 648.546,67 \\ \text { Dia Típico } & 1.183 .803,33 & - & 1.899 .522,00\end{array}$

Estas transações foram realizadas no conjunto de munícipios abrangidos pelo programa de Bilhete Único Estadual. De certo, se trata de viagens tanto de foliões aproveitando o benefício para comemorar o carnaval, quanto de pessoas realizando suas atividades diárias independente da realização do evento na cidade. Para a análise, no entanto, buscou-se entender em que esta festividade influiu nos padrões de deslocamento da Região Metropolitana.

\subsection{Distribuição Modal}

A análise da divisão modal, ou seja, dos modos de transporte utilizados pelos usuários do BUE revela uma semelhança entre o dia de carnaval e o dia típico e uma distinção com o dia de fim de semana, em termos de escolha por linhas de abrangência intermunicipal (52\% do total de transações em um dia de carnaval, contra $51 \%$ em um dia típico e $60 \%$ em um dia de fim de semana) e aquelas de abrangência municipal. Nota-se que as viagens dos "foliões" da Região Metropolitana ficaram concentradas nos modos rodoviários (os ônibus intermunicipais e municipais corresponderam a $60 \%$ das viagens), em níveis semelhantes àqueles observados em um dia típico. Houve um aumento considerável da quantidade de transações no metrô, que passou de $18 \%$ do total em um dia típico e $11 \%$ em um dia de fim de semana para $23 \%$ nos dias de carnaval. Provavelmente, esse fato está ligado ao maior uso deste modo para o deslocamento dentro do município do Rio de Janeiro, em especial ao Centro da cidade. 0 trem apresenta uma queda de utilização nos dias de carnaval, similarmente ao que acontece nos dias de fim de semana. Essa subutilização pode ser explicada principalmente pelo aumento no tempo do intervalo entre os trens e pela operação com parada em todas as estações, o que aumenta o tempo de viagem e reduz a atratividade deste sistema. As barcas apresentaram um expressivo percentual de transações, indicando grande quantidade de passageiros vindo de Niterói para o Rio de Janeiro.

\begin{tabular}{llll}
\multicolumn{4}{c}{ Tabela 3. Distribuição Modal das Transações no Sistema BUE } \\
\hline Modo Utilizado & Carnaval & Fim de Semana & Dia Típico \\
\hline Linhas Intermunicipais & $52 \%$ & $60 \%$ & $51 \%$ \\
Ônibus Intermunicipais & $42 \%$ & $51 \%$ & $41 \%$ \\
Vans Intermunicipais & $4 \%$ & $5 \%$ & $2 \%$ \\
Barcas & $3 \%$ & $1 \%$ & $4 \%$ \\
Trem & $3 \%$ & $3 \%$ & $5 \%$ \\
\hline Linhas Municipais & $48 \%$ & $40 \%$ & $49 \%$ \\
Metrô & $23 \%$ & $11 \%$ & $18 \%$ \\
Ônibus Municipais & $18 \%$ & $23 \%$ & $19 \%$ \\
Trem & $6 \%$ & $5 \%$ & $11 \%$ \\
BRT & $1 \%$ & $1 \%$ & $1 \%$ \\
\hline Total Geral & $100 \%$ & $100 \%$ & $100 \%$
\end{tabular}

\subsection{Distribuição Horária}

As transações com BUE durante os dias de carnaval ocorrem bem distribuídas ao longo do dia, sem que seja possível identificar um pico expressivo. Este comportamento é bem diferente do observado em um dia típico, principalmente. Nos dias de carnaval, foi notado um expressivo aumento das viagens durante a faixa horária da noite e da madrugada (20h às $4 \mathrm{~h}$ ), inclusive em relação aos dias de fim de semana.

\subsection{Distribuição da Origem}

o Rio de Janeiro, na Região Metropolitana, é o município que concentrou a maior quantidade de atrativos para os foliões durante o carnaval. Buscando-se entender a origem dos usuários do Bilhete Único Estadual, foram exploradas as transações em linhas intermunicipais. Foi identificado que Duque de Caxias, Nova Iguaçu, Niterói, São Gonçalo e Belford Roxo são os municípios que concentram, respectivamente, as maiores quantidades de usuários que podem ter vindo ao Rio de Janeiro pular o carnaval, contando com quase $70 \%$ das transações realizadas no sistema de Bilhete Único Estadual.

Algumas mudanças em relação a um dia típico são notadas: uma maior proporção de pessoas vêm de Nova Iguaçu e Belford Roxo durante o carnaval, em comparação com um dia típico, provavelmente dada a proximidade destes municípios em relação ao Rio de Janeiro. Comparativamente, Niterói e São Gonçalo diminuíram em proporção de origem de viagens do BUE, mas continuaram sendo o terceiro e quarto municípios de maior utilização. Em comparação com um dia de fim de semana, Niterói apresentou um aumento da proporção de viagens, o que pode indicar uma maior quantidade de pessoas com destino ao Rio de Janeiro para aproveitar o carnaval.

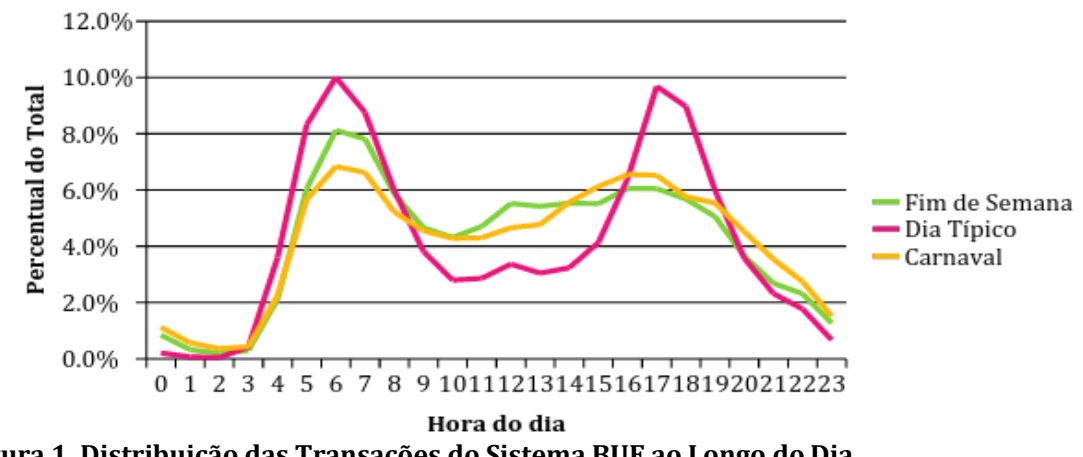

Figura 1. Distribuição das Transações do Sistema BUE ao Longo do Dia 
Tabela 4. Origem na RMRJ de Transações nas Linhas Intermunicipais do Sistema BUE

\begin{tabular}{llll}
\hline Município de Origem & Carnaval & Fim de Semana & Dia Típico \\
\hline Duque de Caxias & $21,9 \%$ & $22,6 \%$ & $22,0 \%$ \\
Nova Iguaçu & $16,7 \%$ & $17,1 \%$ & $15,2 \%$ \\
Niterói & $12,8 \%$ & $10,2 \%$ & $14,7 \%$ \\
São Gonçalo & $9,9 \%$ & $11,2 \%$ & $11,0 \%$ \\
Belford Roxo & $8,5 \%$ & $8,4 \%$ & $7,4 \%$ \\
Outros Municípios & $22,7 \%$ & $23,0 \%$ & $22,6 \%$ \\
\hline
\end{tabular}

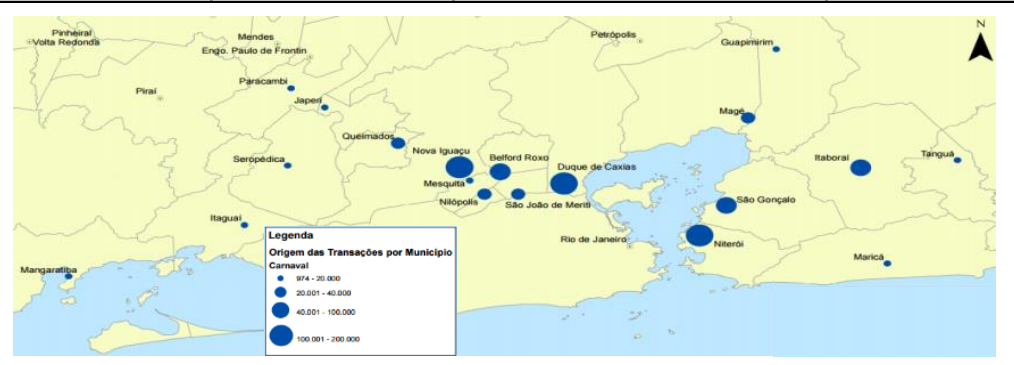

Figura 2. Transações com BUE por Município nos Dias de Carnaval

\section{Conclusão}

O carnaval, assim como qualquer outro grande evento ou feriado em uma cidade, é fator de intensas mudanças no padrão de deslocamentos dos habitantes de uma cidade ou região metropolitana. Em geral, o poder público responsável organiza alguns esquemas táticos para garantir a fluidez de pessoas considerando às limitações de capacidade dos sistemas de transporte de passageiros. Nem sempre, porém, os resultados positivos e negativos do planejamento da oferta de transportes são comparados com sua real utilização. A análise dos dados do sistema de Bilhete Único Estadual revela-se uma importante ferramenta que permite esta análise de forma efetiva e em tempo curto. Este estudou permitiu comparar a quantidade de passageiros que utilizou o sistema, a divisão modal adotada, a sua distribuição ao longo do dia e a origem estimada das viagens. 0 número de viagens no sistema BUE durante os dias de carnaval foi semelhante ao de um dia de fim de semana. Houve, no entanto, diversas particularidades da utilização tanto em relação ao dia de fim de semana quanto ao dia típico. No carnaval, o metrô apresentou crescimento expressivo de utilização em comparação com os outros dias, apesar do modo rodoviário ser o mais utilizado. As viagens foram bem distribuídas ao longo do dia, não havendo picos de demanda tão proeminentes quanto em dias habituais. Houve aumento da utilização durante o período da noite e da madrugada. Alguns municípios como Nova Iguaçu e Belford Roxo tiveram proporcionalmente maior quantidade de origem de transações, talvez pela sua proximidade com a cidade do Rio de Janeiro.

A análise sobre estes dados é apenas uma parte do total de viagens que são empreendidas no sistema de transporte público da Região Metropolitana do Rio de Janeiro. Há, além das viagens realizadas no programa de BUE, as viagens do programa de Bilhete Único Carioca e àquelas que são pagas diretamente em dinheiro ou em cartões unitários. Se fossem analisados os dados para a totalidade do sistema, seria possível estimar a confiabilidade do mesmo em relação à demanda e, em última instância, verificar a validade dos esquemas operacionais montados pelos agentes públicos responsáveis. Para tanto, seria necessário planejar a adoção de um bilhete verdadeiramente único, que abranja todos os benefícios e sistemas existentes na Região Metropolitana e que pudesse ser disponibilizado para o passe semanal, mensal ou anual, ou seja, pudesse ser utilizado indefinidamente no período contratado. Somente assim o bilhete poderá, por um lado, garantir a confiança do cidadão em seu uso e, por outro, englobar para uma eventual análise todas as viagens realizadas pelo mesmo. Mas, até lá, há de se passar alguns carnavais.

\section{Referências}

AGARD, B.; PAROVI NIA, V.; TRÉPANIER, M. Assessing public transport travel behaviour from smart card data with advanced data mining techniques. XIII World Conference on Transport Research. Rio de janeiro: WCTR. 2013. p. 1-13.

CARRERA, F.; FERREIRA JR, J. The Future of Spatial Data Infrastructures: Capacity-building for the Emergence of Municipal SDIs. International Journal of Spatial data Infrastructures Research, Vol. 2 2007. 49-68.

CASTRO, MAGALHÃES DAYAN; DAVIS JR, CLODOVEU A.; MOURA, ANA CLARA M. Infraestrutura de Dados Espaciais para o Plano Diretor de Desenvolvimento Integrado da Região Metropolitana de Belo Horizonte. XXIV Congresso Brasileiro de Cartogrfia. Aracaju: [s.n.]. 2010. p. 1153-1159.

CHU, K. K. A.; SPURR, T.; CHAPLEAU, R. Travel demand characterization for a large urban transit infrastructure using smart card transaction data. WCTR 13th. Rio de Janeiro: [s.n.]. 2013.

DAVIS JR, CLODOVEU AUGUSTO, ALVES, LEONARDO LACERDA. Infra-Estruturas de Dados Espaciais: Potencial para Uso Local. Revista iP, 2007. 1-14.

DUNN, T. P. Role of Technology in Surface Transportation Strategy Development. Portugal: Massachusetts Institute of Technology, 2009.

FRIEDMAN, M. S. et al. Impact of Changes in Transportation and Commuting Behaviors During the 1996 Summer Olympic Games in Atlanta on Air Quality and Childhood Asthma. The Journal of the American Medical Association - JAMA, 21 February 2001. 897-905.

GIUliANO, G. Olympics Transportation System Management Performance Analysis. California: Institute of Transportation Studies, University of California, Irvine., 1985.

GUERRA, A. L.; BARBOSA, H. M.; DE OLIVEIRA, L. K. Estimativa de matriz origem/destino utilizando dados do sistema de bilhetagem eletrônica: proposta metodológica. Transportes, v. 22, p. 26-38, 2014.

GUPTA, S. Understading Transit Travel Behavior: Value Added by Smart Cards (M.Sc. Thesis). Massachusetts Institute of Technology. [S.I.]. 2006.

IBGE. Nota Técnica - Estimativas da população dos municípios brasileiros com data de 1o de julho de 2014, 2015. Disponivel em: <http://www.ibge.gov.br/home/presidencia/noticias/pdf/analise_estimativas_2014.pdf>.

LIU, L. et al. Understanding individual and collective mobility patterns from smart card records: A case study in Shenzhen. International IEEE Conference 12th. [S.l.]: [s.n.]. 2009.

MORENCY, C.; TRÉPANIER, M.; AGARD, B. Measuring transit use variability with smart-card data. Transport Policy, v. $14,2007$. 
MUNIZAGA, M. et al. Validating Travel Behavior Estimated From Smartcard Data. Transportation Research Part C, p. 70-79, 2014.

MUNIZAGA, M.; PALMA, C. Estimation of a disaggregate multimodal public transport Origin-Destination matrix from passive smartcard data from Santiago, Chile. Transportation Research Part C, p. 9-18, 2012.

MUNIZAGA, M.; PALMA, C.; FISCHER, D. Estimation of a Disaggregate Public Transport OD Matrix From Massive Smart Card Data. XV Chileno de Ingeniería de Transporte. Santiago.: SOCHITRAN. 2011. p. 1-15.

NASSI, C. D.; SILVA, A. J. D. O. D.; MURTA, A. L. S. Infraestrutura de Dados Espaciais Local: Proposta para Aplicação em Planejamento de Transportes Público Coletivo - Estudo de Caso do Sistema de Bilhete Único Intermunicipal do Estado do Rio de Janeiro. XVII Congresso Latino Americano de Transporte Público. Equador: CLATPU. 2013. p. 1-11.

PELLETIER, M.-P.; TRÉPANIER, M.; MORENCY, C. Smart card data use in public transit: A literature review. Transportation Research Part C, p. $557-568,2010$.

RIOTUR-RJ. Um carnaval de bons números para o turismo em 2015, 2015. Disponivel em: <http://www.rio.rj.gov.br/web/riotur/exibeconteudo?id=5332140>. Acesso em: 13 maio 2015.

SETRANS-RJ. Plano Diretor de Transportes Urbanos da Região Metropolitana do Rio de Janeiro. Rio de Janeiro. 2014.

SILVA, A. J. O. Bilhete Único Intermunicipal: Proposta de Modelo Utilizando Técnicas de regressão, Sistema de Informações Geográficas, regressão Geograficamente Ponderada e Análise de Componentes Principais. Rio de Janeiro.: Dissertação UFRJ/COPPE/Programa de Engenharia de Transportes., 2013.

SILVA, A. J. O.; NASSI, C. D.; MURTA, A. L. S. Implicações Econômicas, Sociais e Espaciais do Sistema de Bilhete Único - Região Metropolitana/RJ. XXVI Congresso Nacional de Pesquisa e Ensino em Transportes. Joinville/SC: ANPET. 2012. p. 1033-1044.

SILVA, A. J. O.; NASSI, C. D.; MURTA, A. L. S. O Geoprocessamento na Análise do Sistema de Bilhete Único da Região Metropolitana/RJ. VI Simpósio Regional de Geoprocessamento e Sensoriamento Remoto. Aracaju: Geonordeste. 2012. p. 1-6.

ZHAO, J. Estimating a Rail Passenger Trip Origin-Destination Matrix Using Automatic Data Collection Systems. Computer-Aided Civil and Infrastructure Engineering, v. 22, p. 376-387, 2007.

ZOGHI, H.; SIAMARDI, K.; TOLOUEI, M. Impact of 2008 Olympic Games on Urban Transportation and It's Development Strategies in Beijing. International Conference on Computer and Automation Engineering. Bangkok: IEEE. 2009. p. 252-258.

\section{Abstract}

This article focuses on the analysis of urban mobility patterns during the days of carnival city of Rio de Janeiro from data of the Electronic Ticketing System allowance to transport passengers of the State of Rio de Janeiro program , called Ticket State only. The standards in terms of number of transactions, modal distribution and time and spatial distribution of origin are analyzed comparatively to those found on typical weekdays and weekend. The study concludes that the use of the Electronic Ticketing System data enables responsible public officials and operators to better elaborate operational schemes for large events like the carnival. It is recommended to do so, the SBE is the one fact among all the modes and pricing categories.

Keywords: SBE, Demand, Urban Mobility, BUE, Collective Transport 